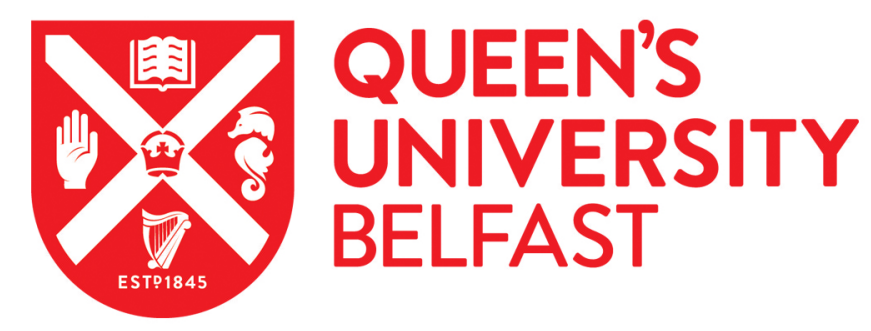

\title{
Magnetic-Field Manipulation of Naturally Occurring Microbial Chiral Peptides to Regulate Gas-Hydrate Formation
}

\author{
Ghaani, M. R., English, N. J., \& Allen, C. C. R. (2020). Magnetic-Field Manipulation of Naturally Occurring \\ Microbial Chiral Peptides to Regulate Gas-Hydrate Formation. Journal of Physical Chemistry Letters, 9079- \\ 9085. https://doi.org/10.1021/acs.jpclett.0c02347
}

Published in:

Journal of Physical Chemistry Letters

Document Version:

Peer reviewed version

Queen's University Belfast - Research Portal:

Link to publication record in Queen's University Belfast Research Portal

Publisher rights

Copyright $\odot 2020$ American Chemical Society. This work is made available online in accordance with the publisher's policies. Please refer to any applicable terms of use of the publisher.

\section{General rights}

Copyright for the publications made accessible via the Queen's University Belfast Research Portal is retained by the author(s) and / or other copyright owners and it is a condition of accessing these publications that users recognise and abide by the legal requirements associated with these rights.

Take down policy

The Research Portal is Queen's institutional repository that provides access to Queen's research output. Every effort has been made to ensure that content in the Research Portal does not infringe any person's rights, or applicable UK laws. If you discover content in the Research Portal that you believe breaches copyright or violates any law, please contact openaccess@qub.ac.uk. 


\title{
Magnetic-Field Manipulation of Naturally-Occurring Microbial Chiral Peptides to Regulate Gas-Hydrate Formation
}

\author{
Mohammad Reza Ghaani,, ${ }^{1, a)}$ Niall J. English ${ }^{1, c)}$ and Christopher C.R. Allen ${ }^{2, b)}$ \\ ${ }^{1}$ School of Chemical and Bioprocess Engineering, University College Dublin, Belfield, \\ Dublin 4, Ireland. \\ ${ }^{2}$ School of Biological Sciences, Queen's University Belfast, University Road, Belfast BT7 \\ $1 N N$, Northern Ireland.
}

\begin{abstract}
Clathrate hydrates are non-stoichiometric crystalline inclusion compounds, wherein a water host lattice entraps small guest molecules in cavities, with methane hydrates the most widespread in Nature. Recent studies have shown that proteins and polypeptides produced by micro-organisms can accelerate methane-hydrate formation. However, the role of magnetic fields and chirality in such phenomena is heretofore unclear. Here, we find primafacie evidence of differently-oriented magnetic fields of varying strength showing intricate control on hydrate-formation kinetics by $\mathrm{R}$ and $\mathrm{S}$ versions of a prototypical aromatic peptide derived from a naturally-occurring, hydrate-promoting. We also discuss the wider implications of these results on chirality in the biosphere and hydrates in the environment.
\end{abstract}

\section{TOC Graphic}

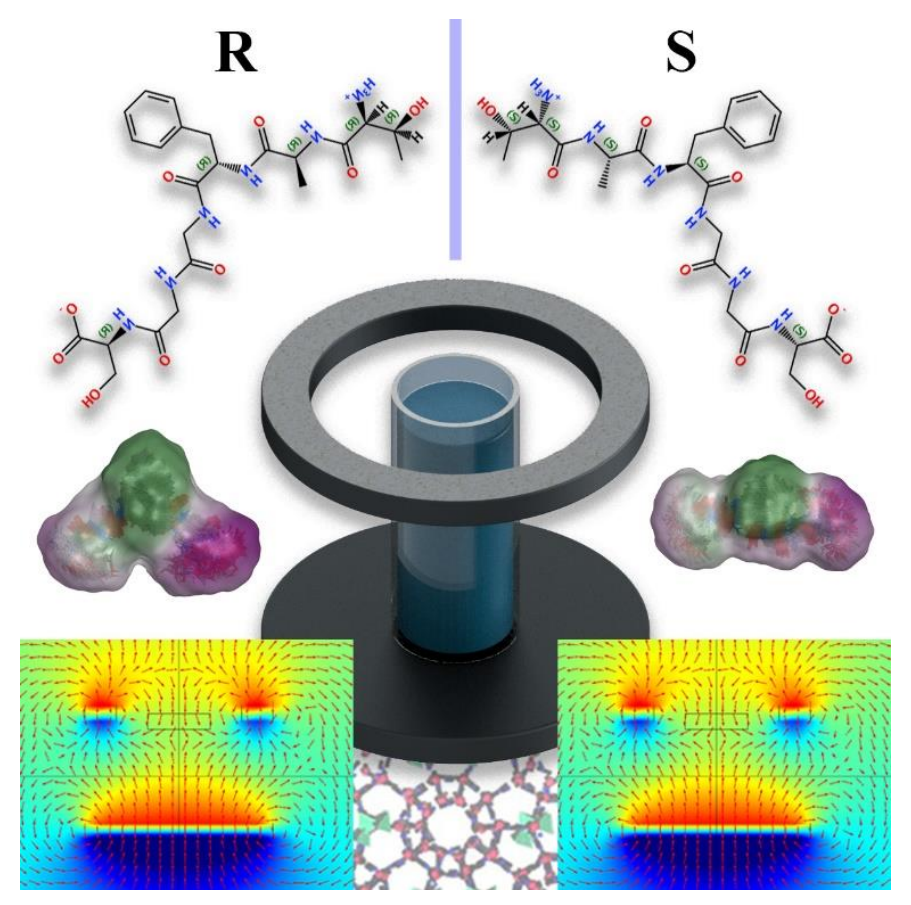

Keywords: Gas hydrates, peptides, microbial, chirality, magnetic fields

Corresponding authors: ${ }^{\text {a) }}$ mohammad.ghaani@ucd.ie, ${ }^{\text {b) }}$ c.allen@ qub.ac.uk, ${ }^{c)}$ niall.english@ucd.ie 
Clathrate hydrates are non-stoichiometric crystalline inclusion compounds in which a water host lattice encages small guest atoms or molecules in cavities ${ }^{1}$. Methane hydrates are the most widespread clathrate in Nature in the permafrost and relatively shallow continental-shelf ocean regions, and constitute the dominant portion of the Earth's bioaccessible carbon reserves ${ }^{2,3}$.

Although it has been known for some time that some proteins (e.g., surfactin $\left.{ }^{4}\right)$ can interact with methane hydrate, and affect its formation kinetics, it has not been clear if such proteins play a role naturally in marine-sediment environments; moreover, in marine sediments, particular materials (e.g., sand) are known to act as hydrate-formation heterogeneousnucleation sites, which may play a greater role in hydrate formation than microbial activity. Recent studies have shown that microorganism-produced proteins and polypeptides (such as an exported, extra-cytoplasmic porin-like protein from a marine methylotroph, GHP1), can accelerate methane-hydrate formation under seafloor-mimicking conditions 5,6

Independently, it was shown that magnetic-field effects can influence both the formation and stability of gas hydrates ${ }^{7}$. Bearing this in mind, this may well provoke the question of how applied magnetic fields might affect chiral systems, i.e., how magneto-chiral effects may dictate hydrate-formation mechanisms, and other important transitions and reactions in (bio-) physical chemistry in general? Certainly, magneto-chiral coupling is well established in molecular dichroism and birefringence vis-à-vis space-inversion and timereversal symmetries (i.e., parity and reversality considerations, respectively), e.g., via analysis of dipole-polarisability tensors ${ }^{7-11}$. Further, in experimental chemical-reaction analysis, the progress of various unrelated reaction pathways involving chiral substrates have been shown to be directly controlled by an applied magnetic field ${ }^{12}$. Critically, the action of magnetic fields on the progress of reaction pathways that utilise catalytic alphacarbon-substituted chiral aromatic moieties is evident ${ }^{13}$. Indeed, the chiral aromatic ring is especially affected by the Lorentz force of an externally-applied magnetic field, because the induced magnetic moment in the aromatic ring interacts sterically with any applied field ${ }^{14}$ - a prime atomistic mechanism for 'amplification' of magneto-chiral effects.

The methylotroph-derived porin-like protein, GHP1, can be used to accelerate the rate of formation of gas hydrates using a possible approximation-catalysis mechanism ${ }^{5}$. This protein incorporates, in an exposed loop, an S-phenylalanine (PHE) moiety that we therefore speculate will interact sterically with an applied magnetic field. From moleculardynamics (MD) modelling (cf. Supporting Information, Fig. S1, as well as ref. 6), we find that this same specific amino acid interacts directly with any methane molecule in its immediate environment ${ }^{6}$. This is through hydrophobic non-covalent bonding, more than other GHP1-surface polypeptide areas - leading ultimately to more catalytic formation of hydrate half-cages by approximation catalysis (cf. Fig. S1(a) and ref. 6). Thus, we hypothesise that the application of an applied magnetic field will affect directly the rate of methane-hydrate formation under substrate-limiting conditions, and that chirality may play a role via magneto-chiral coupling. 
Prior to testing our central hypothesis, we established the effect of an applied magnetic field on the rate of hydrate formation in the absence of peptides/proteins in a model system, shown previously to mimic the temperature and pressure of seafloor conditions in a case of substrate-limited catalytic turnover ${ }^{5}$. Here, we found that there was a measurable decline in the hydrate-formation rate in $\sim 40$ and $\sim 95 \mathrm{mT}$ fields $\left(>90 \% \mathrm{H}_{1}\right.$-confidence on a singletailed Student's t-test) - cf. Fig. S2; the magnetic-field set-up is described in Fig. S3 and Table S1. Subsequent MD-simulation studies have suggested this magnetic-field effect (in the absence of any peptide) can be explained by weakening of hydrogen bonding; ${ }^{15-21}$ indeed, experimentally, the strongest applied field gave an effective $\sim 40 \%$ drop in activity compared to the zero-field case (cf. Fig. S2). Critically, therefore, as we shall see below, we cannot conclude that under these conditions an applied field alone increased hydrateformation rate.

Turning to our key biocatalysis hypothesis, preliminary MD-simulation analysis of the (S-)GHP1 primary sequence revealed a PHE-bearing peptide, as mentioned briefly above, as leading to localised hydrate-formation propensity on the full-GHP1 surface (via approximation catalysis). We codify this peptide as GHP1a; this comprises six amino acids (cf. Fig. S1c). We then tested experimentally addition of R- and S- versions of peptide GHP1a (at a concentration of $6.3 \mathrm{nM}$, cf. Table 1 in 'Methods' - equivalent in molar concentration to the full protein in ref. 5) for its ability to catalyse methane-hydrate formation in variable applied magnetic-field configurations (see Fig. 1). Importantly, we clarify that in order to isolate the putative (field-mediated) bio-catalytic effect, we considered the (relative) measured hydrate-formation rates (subtracting the background, 'water-only' absolute formation rates of Fig. S2); further, we normalised these relative rates vis-à-vis the 'water-only' absolute background rate at that prevailing field condition (e.g., zero-field, 40 and $\sim 95 \mathrm{mT}$ - detailed in Fig. S2). Consequently, we were able to test - independently - both variation of the applied field and inversion of chirality for R- or Sversions of GHP1a (both at $>99 \%$ enantiomeric excess). We were also then able to examine any synergistic and/or competing effects on the catalytic process when both magnetic field and peptide chirality were co-varied. The independently-averaged 'background', wateronly hydrate-formation rate was $0.49 \pm 0.03 \mu \mathrm{mol} . \mathrm{s}^{-1} \mathrm{~cm}^{-3}$, with both GHP1a enantiomers increasing the relative-formation rate by approximately $2.5(\mathrm{~S})$ - to $3.5(\mathrm{R})$ - fold in $\sim 95 \mathrm{mT}$ fields (cf. Fig. 1). There was a marked effect of peptide chirality in the absence of an applied magnetic field - with the highest activity demonstrated by the R-GHP1a; this was statistically significant (>90\% $t$-test $\mathrm{H}_{1}$-confidence) under the conditions used (cf. Fig. 1). However, using an achiral control peptide - comprising 7 linked achiral glycine residues ("polyG control") at the same concentration - a statistically similar increase in hydrateformation rate was observed vis-à-vis S-GHP1a in the absence of a field. Thus, we could not be certain that, in this case, the rate increase was due to biocatalytic effects. However, when the $\sim 95 \mathrm{mT}$ field was applied, an even greater level of statistically significant difference (> 99\% $\mathrm{H}_{1}$-confidence) between relative-formation rates of R-versus S- GHP1a was observed - cf. Fig. 1, with the R-enantiomer having markedly the highest relative 
hydrate-formation rate (2.5 versus 3.5 against the water-only control, with $\mathrm{R}$ the larger). Notably, although the rate of turnover was markedly higher for the $\mathrm{R}$ enantiomer than for a polyG control, the same effect was also observed under $\sim 95 \mathrm{mT}$ field conditions when using the natural $\mathrm{S}$ enantiomer. Profoundly, therefore, an experimentally significant effect of peptide chirality on the hydrate-formation rate was measurable under these conditions when the peptides were incorporated in the presence of an applied magnetic field of sufficient intensity.

(a)

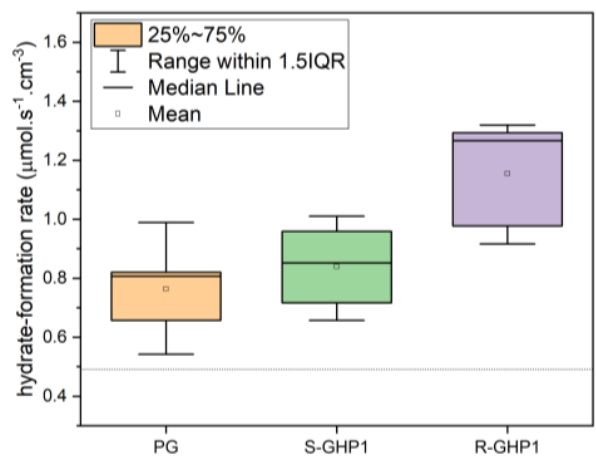

(b)

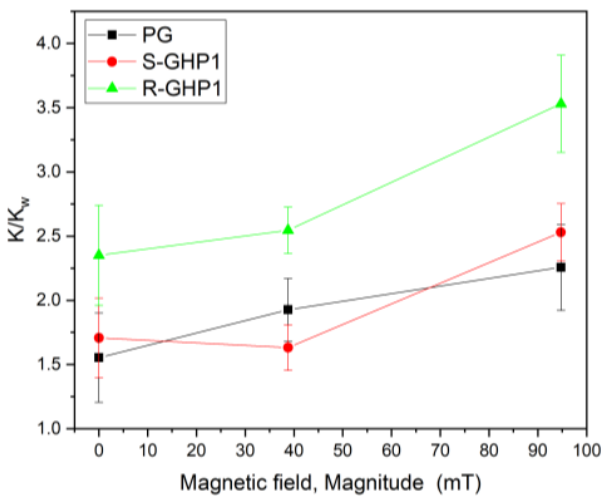

Figure 1. (a) The R-enantiomer shape is a better conformation for relative hydrate-formation rate even without the presence of magnetic field (cf. Fig. 3). The PG and S conformer have similar effects, whilst all three enhance kinetics better than the case with only water (shown by the dotted horizontal line at $0.49 \mu \mathrm{mol} . \mathrm{s}^{-1} \mathrm{~cm}^{-3}$ ). (b) The relative hydrate-formation of part (a) normalised to the field-prevailing background water-only formation rate (cf. Fig. S2) is compared here for R-, S-GHP1a and the PG control for different field intensities; this serves to eliminate the effect of magnetic field on the properties of water.

After considering two different applied field strengths (cf. Fig. S3 and Table S1), an average applied field of $95 \mathrm{mT}$ in the locale of the all-important gas-water interface (whereat the vast majority of hydrate formation was observe to occur) appeared to interact with the 'unnatural' R-enantiomer predominantly. This led to an optimal relative hydrateformation rate, about 1.5 times greater vis-à-vis the $\mathrm{S}$ form of GHP1a (cf. Fig. 1b). When the weaker field of $\sim 40 \mathrm{mT}$ was applied to $\mathrm{S}$ - or R- enantiomer-based catalysis, the relativerate increase was notably less. This suggests that the observed effect may be estimated quantitatively, although is, of course, not necessarily a simple linear response vis-à-vis field strength. However, a more subtle effect of applied-field direction was established between both field strengths, in that the weaker field was oriented in the opposite direction, at $\sim 180^{\circ}$, to the $\sim 95 \mathrm{mT}$ field in proximity to the gas-water interface (see Table S1) - such that this led to a shifting in the level of qualitative R- and S- influence on relative hydrateformation rates (see Fig. 1b). 
(a)

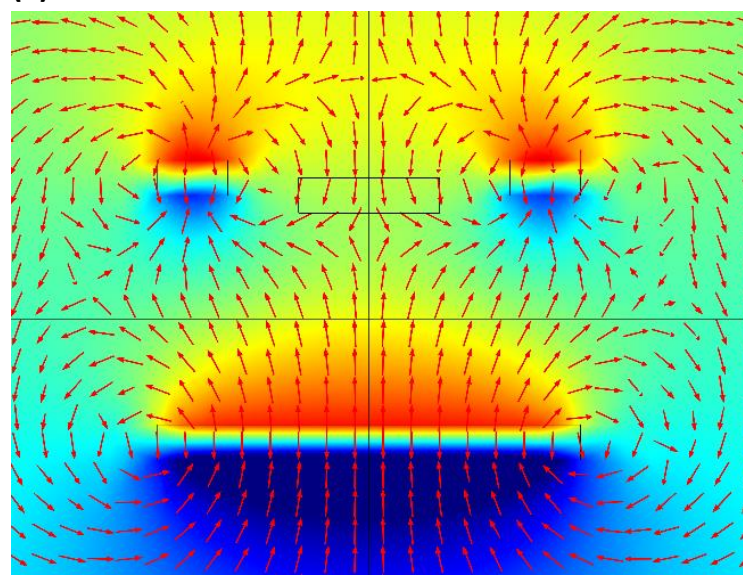

(b)

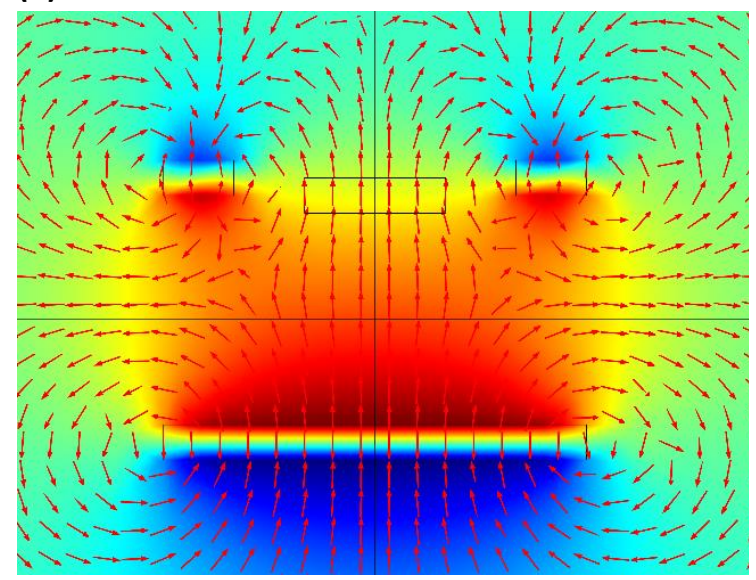

Figure 2. COMSOL-calculated contour plots of the total magnetic-field intensity (see also Fig. S3), with red arrows indicating the field-vector direction and the color map representing field magnitude. The small rectangle in the middle of the upper magnet represents the part of the loaded water which is in the closet contact with gas - where most hydrate formation occurs. (a) 'Opposite poles' of the upper and lower magnet face each other - leading to an 'attraction' between the two magnets. (b) 'Similar poles' face each other, causing repulsion between both magnets. See Fig. S3b and Table S1 for further details.

To elucidate more clearly the microscopic/mechanistic basis for these experimental observations, including field-orientation effects, we next modelled structural changes via non-equilibrium molecular dynamics (NEMD) for either S- and R- GHP1a peptides in contact with methane-supersaturated water systems under field strengths of 0,2 and $5 \mathrm{~T}-$ with both (opposite) field orientations in the case of the 2 and $5 \mathrm{~T}$ fields; this is shown in Fig. 3. It was necessary to use NEMD intensities about an order of magnitude larger than experiment (albeit preserving the $\sim 2.5: 1$ intensity ratio), given that NEMD could only be run for nanoseconds, as opposed to hours. In any event, from NEMD, it can be seen that the positions of residues adjoining the PHE aromatic ring allows for methane to be poised atop the ring. This leads to the water molecules having differing levels of ease of to arrange 'comfortably' around these guest molecules in hydrogen-bonded, half-cage arrangements. Indeed, this alternation of hydro-philic/phobic contacts at the peptides' surfaces appears to underpin the 'scaffolding' for the 'clathrate-anchoring' hypothesis studied with acuity in the context of ice and hydrates at protein surfaces ${ }^{22,23}$ In particular, the more facile halfcage formation is evident for the R-enantiomer under all field conditions (including no applied field), as witnessed experimentally. Moreover, the magnetic field's direction alters subtly the configurational propensity of hydrophilic, hydrogen-bonding contacts for water around the hydrophobic, methane-attracting aromatic centre. The results of Fig. 3 serve to rationalise the greater promoting effect of R-GHP1a relative to the S version, together with the subtle field-orientation effect, in accord with experiment. It should be noted that the field-direction dependence for the S-enantiomer is evident both experimentally (Fig. 1b, 40 vs $95 \mathrm{mT}$, in comparison with zero-field level), and by simulation (Fig. 3b-d, with negative- and positive- directions compared again to the zero-field case) - which supports 
the present study's central hypothesis. Such a trend is less statistically evident for R in the case of both experiment and simulation, but is not unexpected, given its inherently higher (conformation-based) propensity to form hydrates as zero-field conditions.

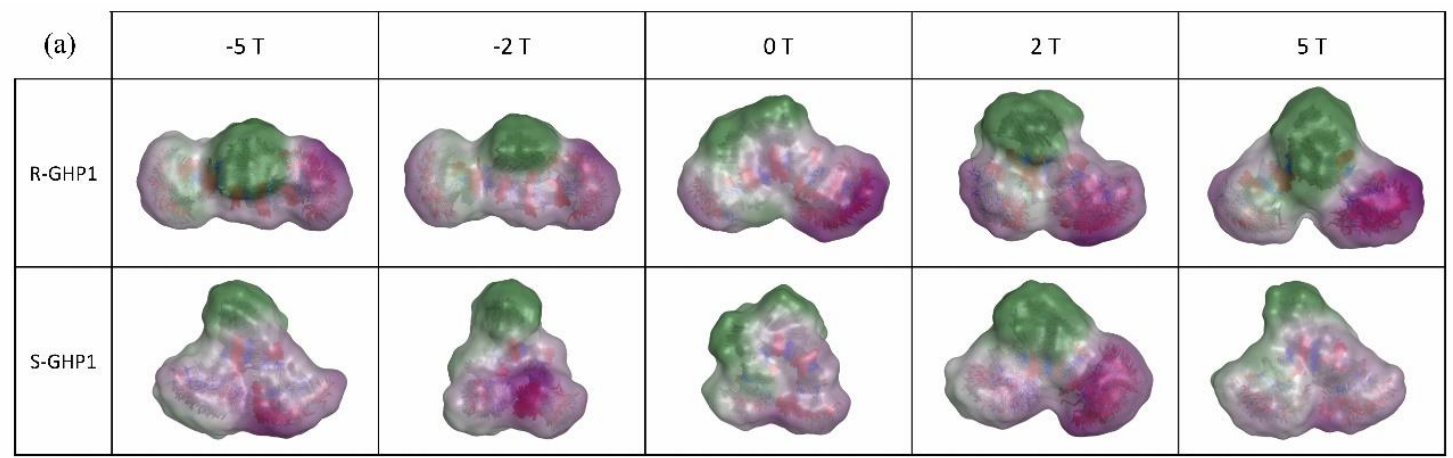

(b)

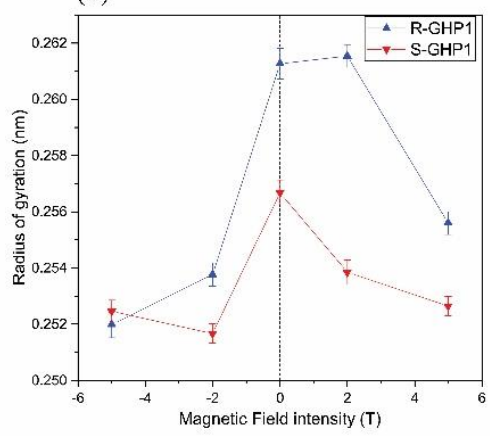

(c)

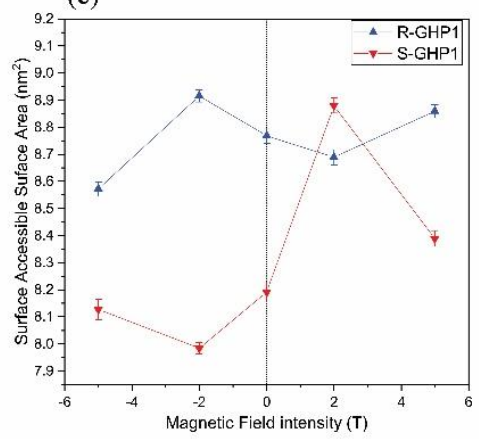

(d)

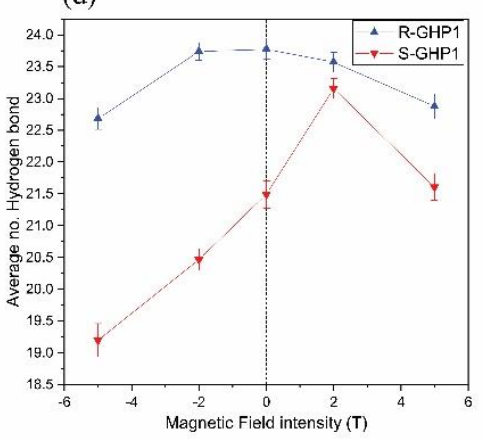

Figure 3. (a) Hydro-philicity and -phobicity around R- and S-GHP1a based on their conformations under different magnetic fields from NEMD simulations at hydrate-forming conditions. The peptide conformation every $50 \mathrm{ps}$ are superposed over $2 \mathrm{~ns}$, with purple denoting hydrophilicity, whilst green represents hydrophobic areas. Characteristic of all peptide conformations is the mutual proximity, and alternation/inter-leaving of hydro-philic and -phobic moieties, which serves to realise pronounced local accumulation of adsorbed water and methane molecules together, thus facilitating hydrate half-cage formation (by de-facto approximation catalysis). A particular case in point for hydrate-cage promotion is the (green) PHE-aromatic centre: here, adsorbed (sometimes long-resident) methane molecules tend to facilitate this. It is clear that the $\mathbf{R}$ form 'stretches' under negative field and the angle between two hydrophilic part becomes lower under positive field. For S-GHP1a, it is 'horseshoe'-shaped in its zero-field conformation, i.e., there are some internal hydrogen bond between parts which keeps this peptide in this shape. Since the magnetic field weakens the hydrogen bonds, in both negative and positive directions, one can see the opening of the peptide. This shape change is less significant in the case of the $S$ form. (b) The application of the magnetic field in both negative and positive directions reduces the radius of gyration of the hydrophobic part of the peptide - the aromatic centre of the PHE residue meaning that the hydrophobic part is less mobile, so the residence time of the adsorbed methane will be higher. (c) The trends in water-accessibility surface area 'echo' clearly that seen in panel (a). (d) The number of hydrogen bonds between the peptide and the surrounding water shows a pronounced decrease upon field exposure, which is due to general effect of magnetic field weakening hydrogen bonds, as mentioned earlier (see also Fig. S2).

Taken together, the present study's overall findings support strongly the hypothesis that an applied magnetic field will moderate the enhancement of hydrate formation in the presence of 
chiral peptides. Because the effect of GHP1a and similar peptides on hydrate is apparently catalytic (and would be expected to apply to hydrate formation of other hydrophobic gases adsorbed at its surface ${ }^{6}$ ), the complementarity of applied magnetic field and peptide catalysis for hydrate formation will not be described by a simple first-order additive effect. Thus, our research might also suggest that an applied magnetic field is likely to have a somewhat unpredictable impact on the control of hydrate formation by peptides and/or proteins in the biosphere. The Earth's magnetic field - about $30 \mu \mathrm{T}$ - will certainly be expected to interact with any aromatic peptides/proteins involved in hydrate stabilisation in Nature. Given this, we cannot therefore rule out the possibility that the previously-documented 'Belfast Hypothesis' - proposing that variations in the Earth's magnetic field affects directly hydrate stability in sediments - could be a key factor influencing global methane turnover when related biotic effects on hydrate stability are considered over geological timescales.

The interaction of magnetic fields with biological entities is well established, but the mechanism of this interaction is not always evident ${ }^{24}$. Our research suggests that such interactions could be explained relatively simplistically through the established interaction of magnetic fields with aromatic amino acids. This means that when we are considering biologically-mediated catalysis, such interactions occur fundamentally at the level of the chiral amino acids found in virtually all proteins.

The findings of the present work complement independently the geophysical implications of varying magnetic fields on the Earth's hydrate deposits and subsequent methane flux to/from the biosphere ${ }^{7}$. Given the climatic importance of gas hydrates, ${ }^{25}$ this may well be important. Presently, the Earth's magnetic field strength is waning, with magnitudes several orders of magnitude lower than used in the present study ${ }^{7}$. If methane-hydrate-bearing sediments are subject to any degree of microbial control, then this same magneto-responsive 'bioamplification' of ambient field effect could be of significance in understanding the wider geophysical implications of microbial activity - including potential climatic effects.

Recent studies have shown that aromatic amino acids are produced using abiotic mechanisms in deep marine sediments ${ }^{26}$. While a number of hypotheses have been presented to explain the origin of chirality in proteins ${ }^{27-32}$, through our findings we can suggest a site for the first appearance of pre-cellular life occurring at the energy-rich methane-hydrate boundary - that would certainly have been present in the methane-rich pre-biotic Earth ${ }^{33,34}$. Indeed, in terms of abiogenesis per se, using the 'chemical-reactor' concept, ${ }^{35}$ it may be the case that "Cold(er)Life" hypotheses, e.g., from carbon sources trapped in sea-ice molecular milieux (eutectic freezing $)^{36}$ or in the ocean's hydrothermal vents ${ }^{37}$ may account for hydrate's possible involvement in terms of facilitating water-methane contact, quite possibly under biological regulation of hydrates (with GHP1 found in the TARA marine-metagenomic database ${ }^{5}$ ).

In any event, the combined effect of the relatively low energy of the Earth's magnetic field being 'bio-amplified' through the action of simple pre-biotic chiral peptides present in ancient sediments, could have resulted in a sufficient evolutionary driving force for the selection of Senantiomer amino acids as the blue-print for chirality in the first biotic proteins. It has been proposed that weak, parity-violating interactions can account for a very small bias favouring 
S- over R- enantiomers in protein secondary structures ${ }^{38}$, which could lead ultimately to an evolutionary bias favouring S-amino acid incorporation into biomolecules when evolutionary drivers over geological timescales are taken into account. However, other studies show that the predominance of S-enantiomer proteins in biological systems is not universal, which might then suggest that other factors come into play ${ }^{39}$. In this study, we have shown quite a substantial differential in the interaction of a structurally simple S- and R- biocatalyst that could, also perhaps over protracted geological timescales, lead to an evolutionary selective pressure favouring R- and/or S- chirality biocatalysts in gas-hydrate-bearing milieux. We propose here that this unravelling of chiral-enzymatic origins is one of the most mechanistically simple, but yet globally profound, examples of biocatalysis occurring in the environment serving as a wonderful test-bed for showing clearly manipulation in Nature by magneto-chiral effects.

\section{METHODS}

$\underline{\text { Hydrate formation and mass-balance-based determination of hydrate-conversion yield }}$

The experimental apparatus for hydrate-formation and dissociation kinetics (as well as estimation of dissociation temperature) employed a pressure vessel fabricated using 316 stainless steel with internal volume of approximately $340 \mathrm{~cm}^{3}$ (see Fig. S3a). A pressure transducer with an uncertainty of $0.02 \mathrm{MPa}$, was used to measure pressure, whilst a thermocouple with an accuracy of $\pm 0.1 \mathrm{~K}$ was inserted into the cell to measure the inner temperature, with temperature/pressure readings every $1 \mathrm{~s}$. The magnetic setup was designed using two N52 Neodymium magnets, the lower magnet was in a disk with $6 \mathrm{~cm}$ diameter and 0.5 thickness and the upper magnet was in ring shape with $6 \mathrm{~cm}$ outer diameter, $4 \mathrm{~cm}$ inner diameter and $0.5 \mathrm{~cm}$ thickness. The water was loaded in a glass tube located in the middle of the setup (Fig S3b). Two different magnetic-field configurations were produced by 'flipping' the upper magnet to have two of the same poles face each other, or the opposite (Fig S3b). Use of an electromagnet coil was not feasible, as localised heating thereat would mitigate against hydrate formation.

Prior to each run, the vessel was sterilised by washing with ethanol solution ( $25 \mathrm{wt} \%)$. Each hydrate-kinetics experiment began with cooling down the main system to the desired temperature of $2.0^{\circ} \mathrm{C}$, via the cooling-water circulating system. Once the system reached the desired temperature, the cell was charged with $10 \mathrm{~cm}^{3}$ of deionised water with the selected amount of reagent (Table 1). The reagents are selected in a way to keep the final solution in the same molar concentration as protein GHP1 which we reported in our previous publication

${ }^{5}$ (Table 1); both R- and S-peptide versions were synthesised to be perfectly enantiomerically pure, and there was no chiral contamination of the water. The cell was evacuated for 3 minutes to remove any residual air, and then pressurised to the desired pressure of 85 bar using pure methane. Due to inevitable Joule-Thomson thermal contraction, the cell pressure was decreased slightly by decreasing the temperature; however, within less than 10 minutes, the temperature stabilised and remains so until the end of the chose hydrate-formation period. Then, a continuous slow pressure decline was observed during the hydrate crystal-growth stage 
(always under the constant-volume conditions), after nucleation. In practice, however, there is some small temperature fluctuation during hydrate formation due to its exothermic nature, but this is countered continually by the temperature-control system (Fig. S3a). Here, the average temperature of the production régime (i.e., the temperature plateau) is considered as the starting temperature of the hydrate-formation process. The system was kept at (or very near) the desired temperature of $2.0^{\circ} \mathrm{C}$ for 3 hours to gauge the final 'molar drop' towards hydrate formation over this period.

Table 1. Concentration of the R- and S- GHP1a peptide and polyglycine (PG). The reagents are selected in a way to keep the final solution in the same molar concentration as the full protein GHP1 which we reported in our previous publication, ref. 5

\begin{tabular}{|c|c|c|c|c|}
\hline Reagent & $\begin{array}{c}\text { Volume } \\
(\mu \mathrm{L})\end{array}$ & MW $(\mathrm{g})$ & Concentration $(\mathrm{mg} / \mathrm{l})$ & Concentration $(\mathrm{nM})$ \\
\hline R-GHP1a & 50 & 1142.38 & 0.21 & 6.32 \\
\hline S-GHP1a & 50 & 1142.38 & 0.21 & 6.32 \\
\hline PG & 50 & 399.365 & 0.13 & 6.32 \\
\hline
\end{tabular}

The 'absorbed number of moles from the gas phase' was determined to track conversion to hydrate formation (which was verified later by measuring temperature discontinuities upon dissociation). This was calculated based on monitoring gas-phase pressure drop continuously on a mass-balance basis. Each experiment repeated 5 times independently to achieve a result with statistical significance. Naturally, the first step in this number-of-gas-phase-moles-frompressure determination lies in defining accurate the compressibility factor, $z$, of the methane ${ }^{40}$ at various pressures and temperatures in the hydrate- formation runs from mass balances on thus-inferred gas-phase-number-of-moles data (from the gas-phase pressure), taking into account the temperature-variation of methane absorption in liquid with literature data for Henry's-Law constants for methane in water ${ }^{41}$.

\section{Molecular-dynamics simulation}

The peptides were placed in $4 \times 4 \times 4 \mathrm{~nm}$ simulation box and solvated with water molecules. The TIP4P model ${ }^{42}$ was used for water, whilst the protein part was parameterised by the OPLS forcefield ${ }^{43}$. The forcefield also combined with the OPLS-UA united-atom model for methane ${ }^{43}$, and replicates the methane-hydrate melting point accurately. The cut-off radius for LennardJones interaction parameters was $1.0 \mathrm{~nm}$, whilst the smooth particle-mesh Ewald method was used to handle long-range electrostatics ${ }^{44}$. The velocity Verlet scheme was used for MD under periodic boundary conditions (PBC) with a time step of $0.5 \mathrm{fs}^{44}$. For extended-system dynamics in the NVT ensemble, a relaxation time of $0.1 \mathrm{ps}$ was applied. All data was sampled over 2-ns production NVT simulations at $275 \mathrm{~K}$ - similar to the experimental conditions. All of the simulations were done using DL-POLY4 ${ }^{45}$. 


\section{Acknowledgements}

M.R.G. thanks Science Foundation Ireland for an Academia-to-Industry Fellowship (17/IFB/5406). CCRA and TS were supported by a QUB AFQCC grant funded by Invest Northern Ireland.

\section{Supporting Information}

Illustration of the selected hydrate-promoting peptide at the surface of the GHP1 protein (Fig. S1). The effect of magnetic field on the absolute methane-hydrate formation rate (Fig. S2). Schematic of gas-hydrate rig and permanent-magnet configurations (Fig. S3). COMSOLcomputed value of the total magnetic field (Table S1) 


\section{References}

(1) Makogon, I. F. Hydrates of Hydrocarbons; Pennwell Books, 1997.

(2) Boswell, R.; Collett, T. S. Current Perspectives on Gas Hydrate Resources. Energy Environ. Sci. 2011, 4 (4), 1206-1215. https://doi.org/10.1039/C0EE00203H.

(3) Kvenvolden, K. A.; Rogers, B. W. Gaia's Breath-Global Methane Exhalations. Mar. Pet. Geol. 2005, 22 (4), 579-590. https://doi.org/10.1016/J.MARPETGEO.2004.08.004.

(4) Rogers, R.; Radich, J.; Xiong, S. The Multiple Roles of Microbes in the Formation, Dissociation and Stability of Seafloor Gas Hydrates.

(5) Ghaani, M. R.; Allen, C. C. R.; Young, J. M.; Nandi, P. K.; Dandare, S. U.; Skvortsov, T.; English, N. J. Microbial Stabilization and Kinetic Enhancement of Marine Methane Hydrates. Geomicrobiol. J. 2020, 37 (3), 279-286. https://doi.org/10.1080/01490451.2019.1695982.

(6) Ghaani, M. R.; Allen, C. C. R.; Skvortsov, T.; English, N. J. Engineering Peptides to Catalyze and Control Stabilization of Gas Hydrates: Learning From Nature. $J$. Phys. Chem. Lett. 2020, 11 (13), 5068-5075. https://doi.org/10.1021/acs.jpclett.0c01224.

(7) English, N. J.; Allen, C. C. R. Magnetic-Field Effects on Methane-Hydrate Kinetics and Potential Geophysical Implications: Insights from Non-Equilibrium Molecular Dynamics. Sci. Total Environ. 2019, 661, 664-669. https://doi.org/10.1016/j.scitotenv.2019.01.041.

(8) Baranova, N.B.; Zel'dovich, B. Ya, Theory of a new linear magnetorefractive effect in liquids, Molec. Phys. 1979, 38, 1085-1098. https://doi.org/10.1080/00268977900102261

(9) Wagnière, G.; Maier, E. The influence of a static magnetic field on the absorption coefficient of a chiral molecule. Chem. Phys. Lett. 1982, 93, 78-81. https://doi.org/10.1016/0009-2614(82)85059-8

(10) Barron, L.D. Molecular Light Scattering and Optical Activity, Cambridge University Press, 1982.

(11) Barron, L.D.; Vrbancich J., Magneto-chiral birefringence and dichroism. Molec. Phys. 1984, 51, 715-730. https://doi.org/10.1080/00268978400100481

(12) Micali, N.; Engelkamp, H.; van Rhee, P. G.; Christianen, P. C. M.; Scolaro, L. M.; Maan, J. C. Selection of Supramolecular Chirality by Application of Rotational and Magnetic Forces. Nat. Chem. 2012, 4 (3), 201-207. https://doi.org/10.1038/nchem.1264.

(13) Kozlova, S. G.; Ryzhikov, M. R.; Kompankov, N. B.; Zavakhina, M. S. Influence 
of Magnetic Field on the Mobility of Aromatic Chiral Molecules. J. Phys. Chem. B 2016, 120 (30), 7517-7521. https://doi.org/10.1021/acs.jpcb.6b05572.

(14) Kondepudi, D. K.; Nelson, G. W. Weak Neutral Currents and the Origin of Biomolecular Chirality. Nature 1985, 314 (6010), 438-441. https://doi.org/10.1038/314438a0.

(15) Zhao, L.; Ma, K.; Yang, Z. Changes of Water Hydrogen Bond Network with Different Externalities. Int. J. Mol. Sci. 2015, 16 (12), 8454-8489. https://doi.org/10.3390/ijms16048454.

(16) Jha, P.; Xanthakis, E.; Jury, V.; Le-Bail, A. An Overview on Magnetic Field and Electric Field Interactions with Ice Crystallisation; Application in the Case of Frozen Food. Crystals 2017, 7 (10), 299. https://doi.org/10.3390/cryst7100299.

(17) Maheshwary, S.; Patel, N.; Sathyamurthy, N.; Kulkarni, A. D.; Gadre, S. R. Structure and Stability of Water Clusters (H 2 O) n , n = 8-20: An Ab Initio Investigation. J. Phys. Chem. A 2001, 105 (46), 10525-10537. https://doi.org/10.1021/jp013141b.

(18) $\mathrm{Su}, \mathrm{N}$.; Wu, C.-F. Effect of Magnetic Field Treated Water on Mortar and Concrete Containing Fly Ash. Cem. Concr. Compos. 2003, 25 (7), 681-688. https://doi.org/10.1016/S0958-9465(02)00098-7.

(19) Toledo, E. J. L.; Ramalho, T. C.; Magriotis, Z. M. Influence of Magnetic Field on Physical-Chemical Properties of the Liquid Water: Insights from Experimental and Theoretical Models. J. Mol. Struct. 2008, 888 (1-3), 409-415. https://doi.org/10.1016/j.molstruc.2008.01.010.

(20) Zhou, K. X.; Lu, G. W.; Zhou, Q. C.; Song, J. H.; Jiang, S. T.; Xia, H. R. Monte Carlo Simulation of Liquid Water in a Magnetic Field. J. Appl. Phys. 2000, 88 (4), 1802-1805. https://doi.org/10.1063/1.1305324.

(21) Wang, Q.; Li, L.; Chen, G.; Yang, Y. Effects of Magnetic Field on the Sol-Gel Transition of Methylcellulose in Water. Carbohydr. Polym. 2007, 70 (3), 345-349. https://doi.org/10.1016/j.carbpol.2007.04.006.

(22) Hudait, A.; Odendahl, N.; Qiu, Y.; Paesani, F.; Molinero, V. Ice-Nucleating and Antifreeze Proteins Recognize Ice through a Diversity of Anchored Clathrate and Ice-like Motifs. J. Am. Chem. Soc. 2018, 140 (14), 4905-4912.

(23) Hudait, A.; Qiu, Y.; Odendahl, N.; Molinero, V. Hydrogen-Bonding and Hydrophobic Groups Contribute Equally to the Binding of Hyperactive Antifreeze and Ice Nucleating Proteins to Ice. J. Am. Chem. Soc. 2019.

(24) Muehsam, D. J.; Pilla, A. A. A Lorentz Model for Weak Magnetic Field Bioeffects: Part I-Thermal Noise Is an Essential Component of AC/DC Effects on Bound Ion Trajectory. Bioelectromagnetics 2009, 30 (6), 462-475. https://doi.org/10.1002/bem.20494.

(25) Ruppel, C. D.; Kessler, J. D. The Interaction of Climate Change and Methane 
Hydrates. Rev. Geophys. 2017, 55 (1), 126-168.

https://doi.org/10.1002/2016RG000534.

(26) Ménez, B.; Pisapia, C.; Andreani, M.; Jamme, F.; Vanbellingen, Q. P.; Brunelle, A.; Richard, L.; Dumas, P.; Réfrégiers, M. Abiotic Synthesis of Amino Acids in the Recesses of the Oceanic Lithosphere. Nature 2018, 564 (7734), 59-63. https://doi.org/10.1038/s41586-018-0684-z.

(27) Rikken, G. L. J. A.; Raupach, E. Enantioselective Magnetochiral Photochemistry. Nature 2000, 405 (6789), 932-935. https://doi.org/10.1038/35016043.

(28) Kuhn, A.; Fischer, P. Absolute Asymmetric Reduction Based on the Relative Orientation of Achiral Reactants. Angew. Chemie Int. Ed. 2009, 48 (37), 68576860. https://doi.org/10.1002/anie.200902841.

(29) Ribo, J. M. Chiral Sign Induction by Vortices During the Formation of Mesophases in Stirred Solutions. Science (80-. ). 2001, 292 (5524), 2063-2066. https://doi.org/10.1126/science.1060835.

(30) Aquilanti, V.; Maciel, G. S. Observed Molecular Alignment in Gaseous Streams and Possible Chiral Effects in Vortices and in Surface Scattering. Orig. Life Evol. Biosph. 2007, 36 (5-6), 435-441. https://doi.org/10.1007/s11084-006-9048-z.

(31) Berger, R.; Quack, M. Electroweak Quantum Chemistry of Alanine: Parity Violation in Gas and Condensed Phases. ChemPhysChem 2000, 1 (1), 57-60. https://doi.org/10.1002/1439-7641(20000804)1:1<57::AID-CPHC57>3.0.CO;2-J.

(32) Flores, J. J.; Bonner, W. A.; Massey, G. A. Asymmetric Photolysis of (RS)Leucine with Circularly Polarized Ultraviolet Light. J. Am. Chem. Soc. 1977, 99 (11), 3622-3625. https://doi.org/10.1021/ja00453a018.

(33) Lovelock, J. E.; Margulis, L. Homeostatic Tendencies of the Earth's Atmosphere. In Cosmochemical Evolution and the Origins of Life; Springer Netherlands: Dordrecht, 1974; pp 93-103. https://doi.org/10.1007/978-94-010-2239-2_8.

(34) Lenton, T.M.; Dutreuil, S.; Latour, B. Life on Earth is Hard to Spot, The Anthropocene Review, 1-25, 2020. https://doi.org/10.1177/2053019620918939

(35) Stüeken, E.E.; Anderson, R.E.; Bowman, J.S.; Brazelton; Colangelo-Lllis, J.; Goldman, A.D., Som, S.M.; Baross, J.A. Did life originate from a global chemical reactor? Geobiol. 2013, 1-26. DOI: 10.1111/gbi.12025

(36) Levy, M., Miller, S.L.; Brinton, K.; Bada, J.L. Prebiotic synthesis of adenine and amino acids under Europa-like conditions, Icarus 2000, 145, 609-613. doi: 10.1006/icar.2000.6365.

(37) de Wit, M.J.; Furnes, H. 3.5-Ga hydrothermal fields and diamictites in the Barberton Greenstone Belt-Paleoarchean crust in cold environments, Science 
Advances 2016, 2, e1500368. DOI: 10.1126/sciadv. 1500368

(38) Mason, S. F.; Tranter, G. E. Energy Inequivalence of Peptide Enantiomers from Parity Non-Conservation. J. Chem. Soc. Chem. Commun. 1983, No. 3, 117. https://doi.org/10.1039/c39830000117.

(39) Shang, Z.; Winter, J. M.; Kauffman, C. A.; Yang, I.; Fenical, W. Salinipeptins: Integrated Genomic and Chemical Approaches Reveal Unusual $\langle\mathrm{scp}\rangle \mathrm{d}</ \mathrm{Scp}\rangle$ Amino Acid-Containing Ribosomally Synthesized and Post-Translationally Modified Peptides (RiPPs) from a Great Salt Lake Streptomyces Sp. ACS Chem. Biol. 2019, 14 (3), 415-425. https://doi.org/10.1021/acschembio.8b01058.

(40) Friend, D. G. Tables for the Thermophysical Properties of Methane; Ely, J. F., Ingham, H., (U.S.), N. I. of S. and T., Eds.; NIST technical note ; 1325.; Boulder, Colo: U.S. Dept. of Commerce, National Institute of Standards and Technology, 1989.

(41) Sander, R. Henry's Law Constants. In NIST Chemistry WebBook, NIST Standard Reference Database Number 69; National Institute of Standards and Technology: Gaithersburg MD, 2018; p 20899.

(42) Hernandes, M. Z.; Da Silva, J. B. P.; Longo, R. L. Chemometric Study of Liquid Water Simulations. I. The Parameters of the TIP4P Model Potential. J. Comput. Chem. 2003, 24 (8), 973-981. https://doi.org/10.1002/jcc.10273.

(43) MacKerell, A. D.; Bashford, D.; Bellott, M.; Dunbrack, R. L.; Evanseck, J. D.; Field, M. J.; Fischer, S.; Gao, J.; Guo, H.; Ha, S.; Joseph-McCarthy, D.; Kuchnir, L.; Kuczera, K.; Lau, F. T. K.; Mattos, C.; Michnick, S.; Ngo, T.; Nguyen, D. T.; Prodhom, B.; Reiher, W. E.; Roux, B.; Schlenkrich, M.; Smith, J. C.; Stote, R.; Straub, J.; Watanabe, M.; Wiórkiewicz-Kuczera, J.; Yin, D.; Karplus, M. AllAtom Empirical Potential for Molecular Modeling and Dynamics Studies of Proteins. J. Phys. Chem. B 1998, 102 (18), 3586-3616. https://doi.org/10.1021/jp973084f.

(44) Scott, R.; Allen, M. P.; Tildesley, D. J. Computer Simulation of Liquids. Math. Comput. 1991, 57 (195), 442. https://doi.org/10.2307/2938686.

(45) Todorov, I. T.; Smith, W.; Trachenko, K.; Dove, M. T. DL_POLY_3: New Dimensions in Molecular Dynamics Simulations via Massive Parallelism. $J$. Mater. Chem. 2006, 16 (20), 1911. https://doi.org/10.1039/b517931a. 